The Astrophysical Journal, 545:L161-L164, 2000 December 20

(C) 2000. The American Astronomical Society. All rights reserved. Printed in U.S.A.

\title{
EMISSION-LINE INTENSITY RATIOS IN Fe XVII OBSERVED WITH A MICROCALORIMETER ON AN ELECTRON BEAM ION TRAP
}

\author{
J. M. Laming ${ }^{1}$ I. Kink, ${ }^{2}$ E. Takacs, ${ }^{3}$ J. V Porto, ${ }^{2}$ J. D. Gillaspy ${ }^{2}$ E. H. Silver, ${ }^{4}$ H. W. Schnopper, ${ }^{4}$ S. R. Bandler, ${ }^{4}$ \\ N. S. Brickhouse, ${ }^{4}$ S. S. Murray, ${ }^{4}$ M. Barbera, ${ }^{5}$ A. K. Bhatia, ${ }^{6}$ G. A. Doschek, ${ }^{1}$ N. Madden, ${ }^{7}$ \\ D. LANDIS, ${ }^{7}$ J. BEEMAN, ${ }^{7}$ AND E. E. HALLER ${ }^{7}$ \\ Received 2000 June 16; accepted 2000 October 11; published 2000 December 6
}

\begin{abstract}
We report new observations of emission line intensity ratios of Fe XVII under controlled experimental conditions, using the National Institute of Standards and Technology electron beam ion trap (EBIT) with a microcalorimeter detector. We compare our observations with collisional-radiative models using atomic data computed in distorted wave and $R$-matrix approximations, which follow the transfer of the polarization of level populations through radiative cascades. Our results for the intensity ratio of the $2 p^{6}{ }^{1} S_{0}-2 p^{5} 3 d^{1} P_{1} 15.014 \AA$ line to the $2 p^{6}{ }^{1} S_{0}-$ $2 p^{5} 3 d^{3} D_{1} 15.265 \AA$ line are $2.94 \pm 0.18$ and $2.50 \pm 0.13$ at beam energies of 900 and $1250 \mathrm{eV}$, respectively. These results are not consistent with collisional-radiative models and support conclusions from earlier EBIT work at the Lawrence Livermore National Laboratory that the degree of resonance scattering in the solar $15.014 \AA$ line has been overestimated in previous analyses. Further observations assess the intensity ratio of the three lines between the $2 p^{6}-2 p^{5} 3 s$ configurations to the three lines between the $2 p^{6}-2 p^{5} 3 d$ configurations. Both $R$-matrix and distorted wave approximations agree with each other and our experimental results much better than most solar and stellar observations, suggesting that other processes not present in our experiment must play a role in forming the Fe XVII spectrum in solar and astrophysical plasmas.
\end{abstract}

Subject headings: atomic data — methods: laboratory — stars: individual (Capella) — Sun: corona — techniques: spectroscopic - X-rays: general

The high elemental abundance of $\mathrm{Fe}$ and the closed shell structure of $\mathrm{Ne}$-like ions cause $\mathrm{Fe}^{+16}$ to be one of the dominant ions in forming emission-line spectra from plasmas with temperatures $\sim 5 \times 10^{6} \mathrm{~K}$. Fe XVII lines will be the dominant lines in most spectra obtained by gratings on Chandra and XMMNewton from a wide variety of objects. For example, in the Capella spectra acquired by Chandra (Brinkman et al. 2000; Canizares et al. 2000), Fe XVII contributes four or five of the six strongest lines observed. In conditions of ionization equilibrium, Fe XVII is unique in providing an electron temperature diagnostic between two sets of these strong lines (Raymond \& Smith 1986). Solar flare observations have long pointed to discrepancies between the observed intensity ratios among the strong emission lines of Fe XVII in the 15-17 $\AA$ region. The Capella spectra referred to above hint that similar problems may exist for astrophysical sources as well. Among the six strong lines arising from transitions between the ground state $2 p^{6}{ }^{1} S_{0}$ and the excited states $2 p^{5} 3 s{ }^{1} P_{1},{ }^{3} P_{1},{ }^{3} P_{2}$, and $2 p^{5} 3 d{ }^{1} P_{1},{ }^{3} D_{1}$, and ${ }^{3} P_{1}$, the strongest line from $2 p^{5} 3 d^{1} P_{1}$ at $15.014 \AA$ often appears diminished in intensity relative to other features. This has led to suggestions that it may be affected by resonance scattering that removes photons pre-

${ }^{1}$ E. O. Hulburt Center for Space Research, US Naval Research Laboratory, Washington, DC 20375.

${ }^{2}$ National Institute of Standards and Technology, 100 Bureau Drive, Gaithersburg, MD 20899.

${ }^{3}$ Massachusetts Institute of Technology, 77 Massachusetts Avenue, 26-239, Cambridge, MA 02139-4307; and University of Debrecen, Debrecen, Bem ter 18/A, H-4026, Hungary.

${ }^{4}$ Harvard-Smithsonian Center for Astrophysics, 60 Garden Street, Cambridge, MA 02138.

${ }^{5}$ Osservatorio Astronomico G. S. Vaiana, Piazza del Parlamento 1, 90134 Palermo, Italy.

${ }^{6}$ Laboratory for Astronomy and Solar Physics, NASA Goddard Space Flight Center, Greenbelt, MD 20771.

${ }^{7}$ Lawrence Berkeley National Laboratory, 1 Cyclotron Road, Berkeley, CA 94720 . dominantly from the line of sight in this transition (Schmelz, Saba, \& Strong 1992; Phillips et al. 1996, 1997; Bhatia \& Kastner 1999; Saba et al. 1999). Furthermore, the overall intensity of the lines from the $2 p^{5} 3 s$ configuration often appears enhanced relative to the intensity theory would predict when compared with the lines from the $2 p^{5} 3 d$ configuration. This has led some authors (U. Feldman 2000, private communication; see Sampson \& Zhang 1987) to suggest that an inner shell ionization of a $2 p$ electron from the Na-like Fe ion plays a role in forming the spectrum, producing $\mathrm{Ne}$-like $\mathrm{Fe}$ in the excited configuration $2 p^{5} 3 s$. Other explanations suggest that strong dielectric recombination occurs (Liedahl et al. 1990) or simply that the theoretical atomic models do not include a sufficient number of excited levels to adequately treat all the radiative cascades. In this Letter we describe initial results of experiments designed to test some of these ideas.

In a study of tokamak spectra of Fe XVII, Phillips et al. (1997) found good agreement between observations and synthetic spectra calculated from models including the lowest 37 fine-structure levels of Fe XVII (i.e., all configurations up to $2 s 2 p^{6} 3 d$ ), including dielectronic and inner shell satellites of Fe XVI. The collisional data used in Phillips et al. (1997) are similar to some of those used here. They are computed in the distorted wave approximation and tabulated in Bhatia \& Doschek (1992). Tokamak plasma sources do not allow the individual atomic processes to be experimentally isolated and studied in detail. More control is possible with the use of an electron beam ion trap (EBIT). Positive ions are trapped in the space charge of an essentially monoenergetic electron beam that also ionizes and excites the ions. The ion charge state is selected by tuning the electron beam energy. In the case of Ne-like ions, almost complete selectivity is possible. Taking advantage of this, Brown et al. (1998) made detailed observations of Fe XVII at beam energies of 850,1150 , and $1300 \mathrm{eV}$. They observed lines originating from levels with principal quantum number $n$ up to 11 . Their mea- 


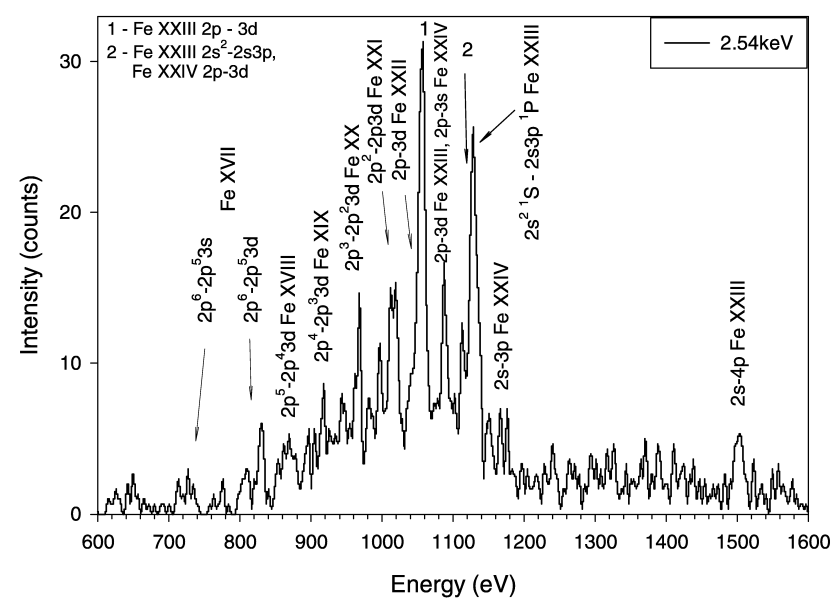

FIG. 1.-Section of a microcalorimeter spectrum of Fe L shell lines recorded at an EBIT beam energy of $2.54 \mathrm{keV}$. Lines from Fe XVII to Fe XXIII are prominent.

surement of the intensity ratio of the strongest $15.014 \AA$ line to the $15.265 \AA$ line originating from the $2 p^{5} 3 d^{3} D_{1}$ level was $3.04 \pm 0.12$, which is in between theoretical values (generally closer to 4) and ratios observed in solar and stellar coronae (usually in the range 2-2.5). Table 2 of Brown et al. (1998) gives a useful summary of the various calculations and observations of this intensity ratio. However, Brown et al. (1998) in their paper report no results for the intensities of the $3 s-3 p$ transitions.

Our experimental technique couples the National Institute of Standards and Technology (NIST) EBIT with a microcalorimeter detector and is discussed in detail in Silver et al. (2000). This offers a combination of high throughput, wide bandpass, and sufficient spectral resolution to allow most lines to be resolved (see Kelley et al. 2000; Silver et al. 2000). One modification from previous experimental runs was to move the microcalorimeter to a new observation port on the EBIT, which allowed much closer access to the trapped ions in the electron beam. This further improved the high-energy bandpass and allowed us to dispense with the X-ray optic. Another important feature is that the microcalorimeter when used in this configuration is polarization blind. Thus, the only correction required for this effect is for the degree of spatial anisotropy in the emission of polarized light; the measured intensity will depend on the degree of polarization through this anisotropy, which is different for lines of different polarization. As we discuss below, such effects are rather small and can be adequately modeled. A part of a spectrum taken at a beam energy of $2500 \mathrm{eV}$ is shown in Figure 1 to illustrate the capabilities of the detector. These data were collected in only 15 minutes. The complete spectrum extends to much higher energies and would include the $\mathrm{Fe} \mathrm{K}$ line complex if the beam energy was sufficiently high. Of course, in this case the Fe L spectrum would not be so strong, and it is these lines, and specifically Fe XVII, that are the main focus of this Letter.

We observed Fe XVII transitions at a variety of electron beam energies between 900 and $4000 \mathrm{eV}$. Comparing spectra at beam energies of 900 and $1250 \mathrm{eV}$ allows us to study what effect (if any) enhanced radiative cascades at the higher energy might have. At $900 \mathrm{eV}$, we are also well below the thresholds for excitation by inner shell ionization of Fe XVI (Sampson \& Zhang 1987) should any be present in our trap (see below). For these two beam energies, experimental intensity ratios were obtained by fitting a sum of Gaussian functions to the experimental data,

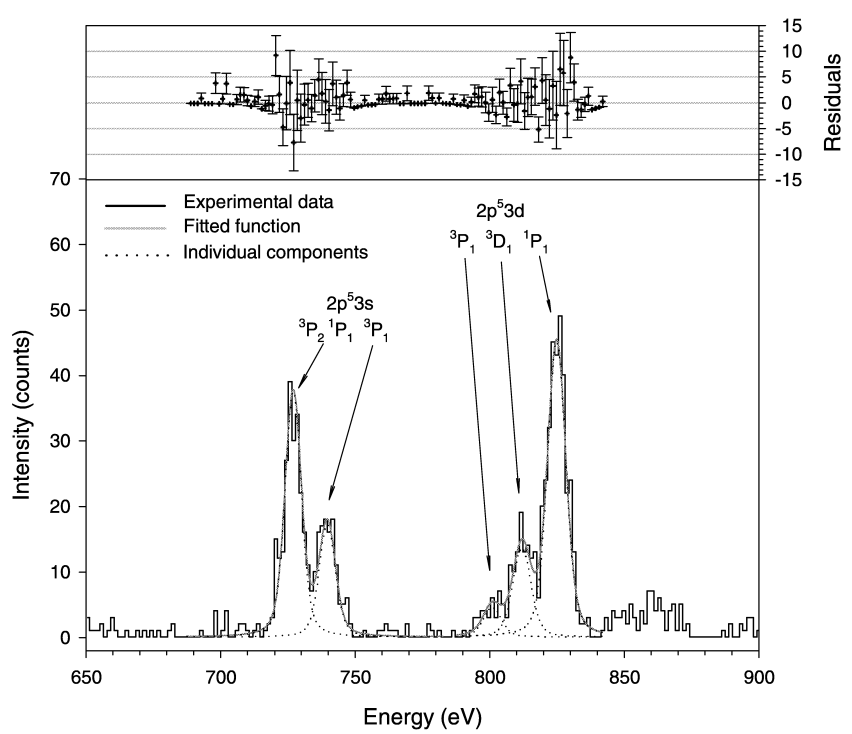

FIG. 2.-Fit to the six strong Fe XVII lines recorded at an electron beam energy of $900 \mathrm{eV}$. The line wavelengths are 15.014, 15.265, 15.456, 16.780, 17.055, and $17.100 \AA$. The last two transitions are blended together at the resolution of the microcalorimeter and are fitted as a single component. The upper panel gives the residuals from the fit.

and the area underneath a particular Gaussian was treated as a line intensity. The Gaussian functions slightly underestimate the "wing" of the spectral lines, and better resemblance is achieved with Voigt functions. However, the results from these different fits remained well within quoted error bars, determined from the statistical quality of the fit (dominant uncertainty) and systematic uncertainties (much smaller contributions) due to the detector/ window efficiency, and therefore the results from Gaussian fits are presented. The data, fit, and residuals for the $900 \mathrm{eV}$ beam energy spectrum are shown in Figure 2. At these beam energies, there is no evidence of inner shell transitions in Fe XVI. The strongest of these lines, the $2 s^{2} 2 p^{6} 3 s^{2} S_{1 / 2}-2 s^{2} 2 p^{5} 3 s^{2}{ }^{2} P_{3 / 2}$, calculated to be at a wavelength between 17.29 and $17.31 \AA$ (Phillips et al. 1997; Bautista 2000), would be easily visible in our spectra, resolved from the Fe XVII 17.055 and $17.100 \AA$ lines. Using higher resolution crystal spectrometers observing one of the Lawrence Livermore National Laboratory EBITs under similar conditions, Brown et al. (1998) also saw no evidence for these transitions.

The spectra were corrected for the transmission efficiencies of the microcalorimeter windows. (The quantum efficiency of the detector is $100 \%$ for energies below $5 \mathrm{keV}$.) The window transmission has been well defined for the entire microcalorimeter bandpass of $0.1-10 \mathrm{keV}$. The three windows are made of polymide $(800 \AA)$ and aluminum (1100 $)$ ), and their thicknesses have been measured to an accuracy of $0.5 \%$ (Powell et al. 1997). The efficiency curve is smooth and featureless in the energy band that includes the Fe $\mathrm{L}$ emission. We have taken great care to minimize potential contamination of these windows, but over long periods of time (days) it is possible that minute amounts of hydrocarbons could collect on the outer window (the most susceptible since it views the vacuum connection to the EBIT). Our calculations show that only several monolayers of nitrogen or oxygen would freeze out on the window given the cleanliness of the EBIT vacuum connection and the extremely low pressures at the initiation of the cooling cycle. Since the Fe XVII lines were measured over a period of 
TABLE 1

Observational and Theoretical Fe xvil Intensities Relative to the 15.014 Å Line

\begin{tabular}{|c|c|c|c|c|c|c|c|c|}
\hline $\begin{array}{c}\text { LINE WAVELENGTH } \\
(\AA)\end{array}$ & $\begin{array}{l}\text { LINE } \\
\text { ENERGY } \\
(\mathrm{eV})\end{array}$ & $\begin{array}{l}\text { UPPER } \\
\text { LEVEL }\end{array}$ & Data $(0.9 \mathrm{keV})$ & \multicolumn{2}{|c|}{ THEORETICAL $(0.9 \mathrm{keV})^{\mathrm{a}}$} & Data $(1.25 \mathrm{keV})$ & \multicolumn{2}{|c|}{ TheORETICAL $(1.25 \mathrm{keV})^{\mathrm{a}}$} \\
\hline 15.265 & 812.5 & $2 p^{5} 3 d^{3} D_{1}$ & $0.34 \pm 0.02^{\mathrm{b}}$ & 0.25 & 0.25 & $0.40 \pm 0.02$ & 0.26 & 0.26 \\
\hline 16.780 & 739.1 & $2 p^{5} 3 s^{3} P_{1}^{\mathrm{c}}$ & $0.45 \pm 0.04$ & $0.46-0.41$ & $0.47-0.41$ & $0.40 \pm 0.02$ & $0.51-0.44$ & $0.47-0.42$ \\
\hline 17.055 & 727.1 & $2 p^{5} 3 s^{1} P_{1}^{\mathrm{c}}$ & & $0.54-0.47$ & $0.60-0.53$ & & $0.58-0.50$ & $0.55-0.47$ \\
\hline $17.100 \ldots \ldots \ldots$ & 725.0 & $2 p^{5} 3 s^{3} P_{2}$ & & $0.34-0.30$ & $0.43-0.38$ & & $0.41-0.35$ & $0.39-0.33$ \\
\hline
\end{tabular}

${ }^{a}$ Theoretical range goes from unpolarized to maximum polarization case. $R$-matrix cross sections are from Mohan et al. 1997 substituted for first 26 excited levels.

${ }^{\mathrm{b}}$ Uncertainties correspond to $1 \sigma$.

${ }^{\mathrm{c}}$ Some authors swap the $L S$ coupling notation for these levels, with $16.780 \AA$ originating from ${ }^{1} P_{1}$ and $17.100 \AA$ from ${ }^{3} P_{1}$.

${ }^{\mathrm{d}}$ Experimental result is for the sum of 17.055 and 17.100 intensities; theoretical results are for each line separately and their sum.

$1 \mathrm{hr}$ (as opposed to several days), the chances of a contamination buildup occurring is unlikely. Furthermore, we point out that the Fe XVII line ratios measured by our instrument are relatively insensitive to even a large amount of contamination. For example, $3000 \AA$ of nitrogen or oxygen ice formed on the outer cryostat window could reduce transmission by $25 \%$ but only alter the ratios for the $3 s / 3 d$ lines by $9 \%$; the $2 p-3 d$ line ratios would remain unchanged. This scenario is highly unlikely since $3000 \AA$ of $\mathrm{N}$ or $\mathrm{O}$ ice is 2 times thicker than the window itself and would undoubtedly break it.

We compare our observations with theoretical results computed using a model $\mathrm{Fe}$ XVII ion comprising the configurations up to $2 s 2 p^{6} 4 d$, i.e., 73 levels, with extra configurations $2 s^{2} 2 p^{5} n s, 2 s^{2} 2 p^{5} n d$, and $2 s 2 p^{6} n p$ for $n=5$ and 6 included for their radiative cascades. This brings the total up to 113 levels. Excitation rates are calculated for monoenergetic electron beams between 0.9 and $4.0 \mathrm{keV}$ energy, including only those levels that are energetically accessible in each case. The calculations use impact excitation cross sections computed in a distorted wave approximation as described in Bhatia \& Doschek (1992) for all transitions among the lowest 73 levels. As an alternative, $R$ matrix cross sections from the ground state to the configurations up to $2 p^{5} 3 d$ (i.e., the first 26 excited levels) can be substituted from Mohan, Sharma, \& Eissner (1997). Energy levels and radiative decay rates are taken from the distorted wave target calculation, supplemented with those for higher lying configurations taken from a computation with the HULLAC (Hebrew University Lawrence Livermore) Code for Atomic Physics (M. Klapisch, A. Bar Shalom, W. H. Goldstein, E. Meroz, A. Chon, \& M. Cohen 1988, unpublished; Goldstein et al. 1988). Collisional excitation rates to these higher levels are scaled from those for the lower lying ones.

For the purposes of modeling emission from the EBIT, we follow the analysis in Takacs et al. (1996) and expand our 113level model to the 457 magnetic sublevels present. This is done in order to account for the polarization arising from the excitation by the electron beam. For the upper levels of the strongest transitions (i.e., those with $j=1$ ), excitation cross sections to individual magnetic sublevels are given in the relativistic distorted wave approximation by Zhang, Sampson, \& Clark (1990). For transitions where a nonrelativistic approach is valid, these data are supplemented with simple results from a Coulomb-Bethe approximation.

All levels with $j \geq 3$ are assumed to be unpolarized. More accurate $m_{j}$ dependent cross sections will be substituted as they become available, but the current procedure should model the transmission of polarization through radiative cascades suffi- ciently accurately to allow us to evaluate the anisotropy of the emission from the EBIT. More precise work would be necessary with crystal spectrometers.

In Table 1 we compare our experimental Fe XVII line intensity ratios with those calculated by the methods outlined above for beam energies of 0.9 and $1.25 \mathrm{keV}$. The theoretical results come from the distorted wave cross sections calculated herein and from the same distorted wave results but with the cross sections for excitation from the ground state to the first 26 excited levels replaced by the $R$-matrix results of Mohan et al. (1997). The range of theoretical ratios extends from results calculated in the limit of zero polarization to those in the limit of maximum polarization. The finite distribution of pitch angles in the electron beam causes the true theoretical result to lie somewhere between these two limits. This depolarization can be estimated from results given in Gu, Savin, \& Beiersdorfer (1999). Even in the limit of no depolarization, the polarization correction required for the microcalorimeter is rather small, less than would be the case for a crystal spectrometer, but remains the dominant systematic uncertainty in our experiment at these two beam energies.

Brown et al. (1998) measured intensity ratios for the 15.014/ $15.265 \AA$ lines of $2.93 \pm 0.16,3.15 \pm 0.17,2.77 \pm 0.19$, and $3.00 \pm 0.20$ from observations at beam energies of 1150, 1150, 850 , and $1300 \mathrm{eV}$, respectively. They then average these values to give a mean result of $3.04 \pm 0.12$, compared with theoretical predictions of around 4. Explicit in this argument is an assumption that the polarizations of these two lines are the same, since the observations were made with crystal spectrometers at Bragg angles where they are polarization sensitive. Such an assumption is supported by theory (Zhang et al. 1990). However, the result obtained using this assumption demonstrates that this same theory gives an intensity ratio between these two lines inconsistent with experiment. While it is quite possible that the calculations get the polarizations right and the intensity ratio wrong, it is certainly not guaranteed. The microcalorimeter employed in the present work is not polarization sensitive, and our experimental values for the intensity ratio- $2.94 \pm 0.18$ at $0.9 \mathrm{keV}$ and $2.50 \pm 0.13$ at $1.25 \mathrm{keV}$-are more robust, being sensitive only to the possible difference in the anisotropy of the emitted radiation. The first value is consistent with the average result of Brown et al. (1998), while the second is slightly lower. These results support the suggestion of Brown et al. (1998) that the effect of resonance scattering in the $15.014 \AA$ resonance line in solar spectra has hitherto been overestimated.

Intensity ratios from our data for the three lines originating from $2 p^{5} 3 s$ to those from $2 p^{5} 3 d$ are shown in Figure 3. For energies greater than $1.25 \mathrm{keV}$, they are obtained by summing 


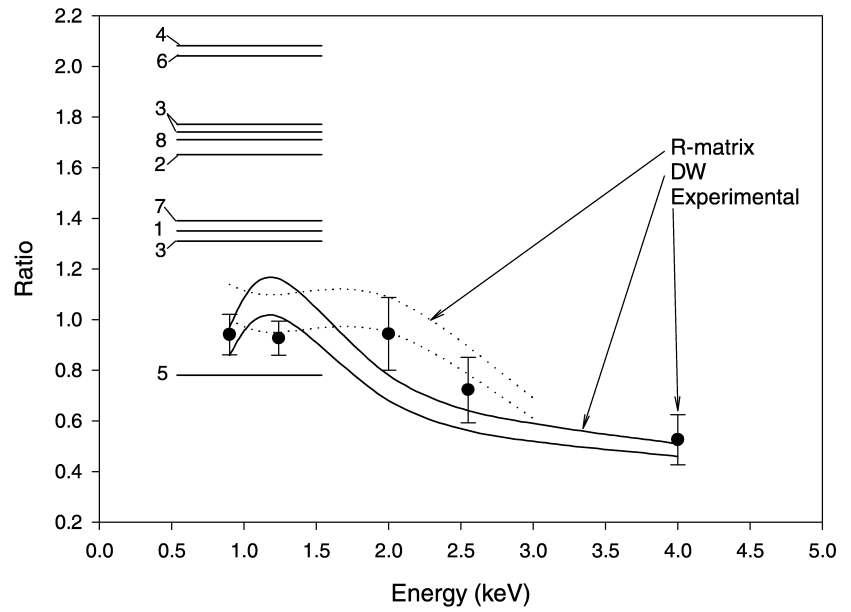

FIG. 3.-Variation of the intensity ratio $\left(I_{16.780}+I_{17.055}+I_{17.100}\right) /\left(I_{15.014}+\right.$ $\left.I_{15.265}+I_{15.456}\right)$ with electron beam energy. The first three lines originate in the $2 p^{5} 3 s$ configuration and the second three in the $2 p^{5} 3 d$. For comparison, results from model calculations using the distorted wave results (see Bhatia \& Doschek 1992) are shown, and where appropriate the $R$-matrix results of Mohan et al. (1997) are overlaid. The range given for each calculation corresponds to the limits of unpolarized (upper curve) and completely polarized (lower curve) emission. The observational ratios from various solar and stellar observations are shown for reference on the same plot. For these, the energy scale on the $x$-axis should be disregarded. Fe XVII is generally formed in plasmas at a temperature of $5 \times 10^{6} \mathrm{~K}$, and electrons with energies close to threshold, i.e., less than $1 \mathrm{keV}$, dominate the excitation rate. The references are (1) Blake et al. (1965); (2) Parkinson (1975); (3) Hutcheon, Pye, \& Evans (1976); (4) McKenzie et al. (1980); (5) Phillips et al. (1982); (6) Acton et al. (1985); (7) Brinkman et al. (2000); and (8) Canizares et al. (2000).

the number of detected photons over the spectral regions where the $2 p^{6}-2 p^{5} 3 s$ and $2 p^{6}-2 p^{5} 3 d$ lines appear, respectively, correcting for the presence of the Fe XIX lines at 15.114 and $15.210 \AA$ ( 820 and $815 \mathrm{eV}$, respectively), by measuring the strong Fe XIX $2 p^{4}-2 p^{3} 3 d$ lines at $13.465,13.507$, and $13.521 \AA$ (920 $\mathrm{eV}$ ) and subtracting off the appropriate ratio determined from calculations with HULLAC. Since Fe XIX has a ${ }^{3} P_{2}$ ground-state, polarization corrections are negligible. The calculated ratios, in the range $0.25-0.33$ for beam energies $2-4 \mathrm{keV}$, also agree well with higher resolution observations of solar active regions (recently reassessed by Phillips et al. 1999). The error bars are estimated from the Poisson counting statistics and uncertainties arising from background subtraction (i.e., the Fe XIX lines) and in the detector/window quantum efficiency but are dominated by a $\pm 20 \%$ error in the measured intensity of the Fe XIX blend at $920 \mathrm{eV}$. Also shown in Figure 3 are observational ratios of the three $3 s$ lines to the three $3 d$ lines taken from various solar and stellar observations.

The experimental ratios for the electron beam energy of 0.9 $\mathrm{keV}$ suggest that the models including the $R$-matrix cross sections are less preferable than those using only distorted wave cross sections. The former cross sections appear to overestimate slightly the emission in the 17.055 and $17.100 \AA$ lines. Elsewhere we have a small preference for the $R$-matrix results, although we caution here that a small contribution to the Fe XVII emission may arise by recombination from $\mathrm{Fe}^{+17}$ into excited states of Fe XVII, and until this effect is adequately modeled firm conclusions might be premature. Our experience with similar spectra of Kr XXVII (Kink et al. 2000) suggests that the inclusion of recombination would increase the theoretical $3 s / 3 d$ ratios. More importantly, the difference between the two theoretical approaches and our experiment is significantly smaller at all energies than the discrepancies between the solar and astrophysical observations summarized in Figure 3 (with the exception of Phillips et al. 1982) and either theory. The basic electron impact excitation theory for these lines in Fe XVII appears to be correct, in contrast with that for the 15.265/15.014 ratio (see Table 1), and one must look to other processes to model the solar and astrophysical observations satisfactorily. These may invalidate the electron temperature diagnostic of Raymond \& Smith (1986).

These measurements are a subset of a larger survey of spectroscopy experiments performed with the microcalorimeter on the NIST EBIT. Future work will concentrate on acquiring spectra with significantly higher statistical quality, simply by increasing the integration time at each beam energy. This might also allow the simultaneous observation of radiative recombination features from the EBIT.

A. K. B., G. A. D., and J. M. L. were supported by NASA contract W19539 (Applied Information Systems Research Program); G. A. D. and J. M. L. were also supported by the NRL/ ONR Solar Magnetism and the Earth's Environment 6.1 Research Option. E. H. S., H. W. S., and S. R. B. acknowledge support in part by NASA grant NAG5-5104, and I. K. acknowledges support from the Swedish Foundation for Cooperation in Research and Higher Education (STINT). We also acknowledge the comments of an anonymous referee, particularly in regard to the Fe XIX lines.

\section{REFERENCES}

Acton, L. W., et al. 1985, ApJ, 291, 865

Bautista, M. A. 2000, J. Phys. B, 33, 71 (erratum 33, 2833)

Bhatia, A. K., \& Doschek, G. A. 1992, At. Data Nucl. Data Tables, 52, 1

Bhatia, A. K., \& Kastner, S. O. 1999, ApJ, 516, 482

Blake, R. L., Chubb, T. A., Friedman, H., \& Unizicker, A. E. 1965, ApJ, 142,

Brinkman, A. C., et al. 2000, ApJ, 530, L111

Brown, G. V., Beiersdorfer, P., Liedahl, D. A., Widman, K., \& Kahn, S. M. 1998, ApJ, 502, 1015 (erratum 532, 1245 [2000])

Canizares, C. R., et al. 2000, ApJ, 539, L41

Goldstein, W. H., Oreg, J., Zigler, A., Bar Shalom, A., \& Klapisch, M. 1988, Phys. Rev. A, 38, 1797

Gu, M. F., Savin, D. W., \& Beiersdorfer, P. 1999, J. Phys. B, 32, 5371

Hutcheon, R. H., Pye, J. P., \& Evans, K. D. 1976, MNRAS, 175, 489

Kelley, R. L., et al. 2000, Nucl. Instrum. Methods Phys. Res., 444, 170

Kink, I., et al. 2000, Phys. Rev. E., submitted

Liedahl, D. A., Kahn, S. M., Osterheld, A. L., \& Goldstein, W. H. 1990, ApJ, 350, L37

McKenzie, D. L., Landecker, P. B., Broussard, R. M., Rugge, H. R., Young, R. M., Feldman, U., \& Doschek, G. A. 1980, ApJ, 241, 409
Mohan, M., Sharma, R., \& Eissner, W. 1997, ApJS, 108, 389

Parkinson, J. H. 1975, Sol. Phys., 42, 183

Phillips, K. J. H., et al. 1982, ApJ, 256, 774

Phillips, K. J. H., Greer, C. J., Bhatia, A. K., Coffey, I. H., \& Keenan, F. P. 1997, A\&A, 324, 381

Phillips, K. J. H., Greer, C. J., Bhatia, A. K., \& Keenan, F. P. 1996, ApJ, 469, L57

Phillips, K. J. H., Mewe, R., Harra-Murnion, L. K., Kaastra, J. S., Beiersdorfer, P., Brown, G. V., \& Liedahl, D. A. 1999, A\&AS, 138, 381

Powell, F. R., et al. 1997, Proc. SPIE, 3113, 432

Raymond, J. C., \& Smith, B. W. 1986, ApJ, 306, 762

Saba, J. L. R., Schmelz, J. T., Bhatia, A. K., \& Strong, K. T. 1999, ApJ, 510, 1064

Sampson, D. H., \& Zhang, H.-L. 1987, Phys. Rev. A, 36, 3590

Schmelz, J. T., Saba, J. L. R., \& Strong, K. T. 1992, ApJ, 398, L115

Silver, E., et al. 2000, ApJ, 541, 495

Takacs, E., et al. 1996, Phys. Rev. A, 54, 1342

Zhang, H. L., Sampson, D. H., \& Clark, R. E. H. 1990, Phys. Rev. A, 41, 198 